\title{
An integral equation approach to orientational phase transitions in two and three dimensional disordered systems
}

\author{
E.Lomba ${ }^{1}$, F.Lado ${ }^{2}$, J.J.Weis ${ }^{3}$ \\ 1 Instituto de Química Física Rocasolano, CSIC, \\ Serrano 119, E-28006 Madrid, Spain \\ 2 Department of Physics, North Carolina State University, \\ Raleigh, North Carolina 27695-8202, USA \\ 3 Laboratoire de Physique Théorique, Bâtiment 210, Université de \\ Paris-Sud, 91405 Orsay Cedex France
}

\begin{abstract}
Received August 1, 2000
The use of inhomogeneous Ornstein-Zernike equations to analyze phase transitions and ordered phases in magnetic systems is explored both in bulk three dimensional disordered Heisenberg systems and in a simple model for a two dimensional ferrofluid monolayer. In addition to closures like the Mean Spherical Approximation, Hypernetted Chain and Zerah-Hansen approximation, the inhomogeneous Ornstein-Zernike equation must be complemented by a one-body closure, for which the Born-Green equation has been used in this paper. The results obtained prove that the proposed approach can furnish accurate estimates for the paramagneticferromagnetic transition in the three dimensional Heisenberg spin fluid, reproducing reliably the structure of the isotropic and ordered phases. In two dimensions, the results are fairly accurate as well, both for the dipolar film alone and in the presence of external perpendicular fields. At high densities/dipole moments the equation seems to predict a transition to a phase in which the dipoles lie mostly in the plane and are aligned into vortex-like structures. Evidence of this new phase is found in the simulation at somewhat higher couplings.
\end{abstract}

Key words: integral equations, ferromagnetic transitions, antiferromagnetism, dipolar films

PACS: $61.20 . G y, 68.15 .+e, 75.10 .-b, 75.30 .-m$

\section{Introduction}

Orientational phase transitions in topologically disordered magnetic materials have attracted a considerable interest over the years. Depending on the dominant 
type of interaction, however, the experimental information is unevenly available. Thus, in the case of leading magnetic dipole-dipole interactions, ferrofluids have long been studied in detail [1]. On the contrary, the quest for liquid ferromagnetic phases led by exchange interactions remained open until the advent of magnetic levitation techniques [2]. Although evidence had already been found that $\mathrm{Co} / \mathrm{Au}$ melts exhibited a disordered paramagnetic phase [3], the systems involved lied more properly in the glassy domain. Only recently [2], Albrecht and coworkers managed to undercool a $\mathrm{Co}_{80} \mathrm{Pd}_{20}$ melt under its Curie line at zero field levitated in an electromagnetic container in order to avoid nucleation. This type of experiences have renewed the interest to the Heisenberg spin fluid, which is the simplest model that can give a reasonable account of these phase transitions.

But not only bulk systems have attracted the interest of experimentalists, a great deal of work has been devoted to magnetically active films. This field has been particularly active given the progress in epitaxial growth techniques. In this way, a variety of systems have been manufactured by deposition of thin films (a few atomic layers) of paramagnetic particles (metal oxides) on solid (usually metallic) surfaces [4]. The leading interactions here are the Heisenberg spin-spin exchange and spin-orbit coupling with the substrate, which gives rise to a field-like term known as surface anisotropy. Nonetheless, these materials, though disordered, should more properly be considered as two-dimensional glasses. On the other hand, two-dimensional magnetic fluid systems have also recently been studied by Zahn, Mendez-Alcaraz and Maret $[5,6]$ who prepared a two-dimensional colloid by means of an arrangement of superparamagnetic colloidal particles (doped with $\mathrm{Fe}_{2} \mathrm{O}_{3}$ ) confined to a water/air interface. The behaviour of this system is controlled by the magnetic dipole-dipole interaction and can thus be considered an 2D ferrofluid.

In this context, it is not surprising that theoretical investigations have also flourished in the last years. Particularly, renewed effort has been devoted to devise solution methods for the inhomogeneous Ornstein-Zernike equation [7], being evident that the classical isotropic homogeneous integral equation approaches could never throw light into the problem of the structured phases despite its ability to delimit the boundaries of stability in various relevant systems, such as the dipolar hard sphere fluid $[8,9]$ and the ferromagnetic [10] and antiferromagnetic Heisenberg spin fluid [11]. Computer simulation [12-15] and density functional theory $[12,16]$ were for some time the only available alternative to study the thermodynamics and structure of the orientationally ordered phases, until Sokolovska [17] presented the solution of the Mean Spherical Approximation (MSA) for the continuum ferromagnetic phase of the Heisenberg spin fluid, using Lovett's equation [18] to relate the one and two body correlation functions. Independently, Lado and Lomba [19] solved a reference Zerah-Hansen approximation for the same system, but using the Born-Green equation to connect one and two-body density correlations. In fact, Zhong and Petscheck [20], had already solved the Percus-Yevick(PY) approximation for a nematic fluid, but the assumption of rotational invariance on the Ward identity in the zero field limit, introduced a completely uncontrolled approximation in their formalism [21]. A more thorough study of the structured phases of the Heisenberg fluid was later 
presented by Lado, Lomba and Weis [22] and Perera [23]. The main difference between these works lies in the fact that Lado and coworkers made use of an expansion of the correlation function in terms of special orthogonal polynomials constructed using the one body density correlation as weight function. This approach, inspired on Lado's treatment of internal degrees of freedom [24], renders a set of equations for the anisotropic fluid which is formally identical to that of isotropic systems. Aside from this computational aspect, Lado and coworkers [19,22] use the BG equation as one-body closure, while Perera [23] uses Lovett's equation. Whereas both relations are exact, the final results are not necessarily equivalent since the approximations introduced via the two body closure will certainly propagate differently through each one particle equation. More recently, Holovko and Sokolovska [21] have also presented the solution of the MSA for the nematic phase of a simple separable potential model using Lovett's equation as one particle closure. On the other hand, dipolar fluids have proven more cumbersome to tackle, given the theoretical difficulties associated with the long range of the dipolar interaction, and the additional complication derived from dependence of the potential on the orientation of the interparticle vector, but also quite recently Klapp and Patey [25] have presented the solutions of the Reference Hypernetted Chain (RHNC) equation and the MSA for the dipolar hard sphere ferroelectric phases, using both Lovett's and the BG one particle closures. Along the same lines, Lomba, Lado and Weis [26] have also considered a two-dimensional ferrofluid formed by dipolar hard spheres lying on a surface. Here, anisotropy results from the constraint imposed on the dipolar spheres, which although able to freely rotate in space, must have their centers situated in the plane, and from the presence of external fields. Spontaneous symmetry ruptures, though sought, do not seem to occur in this system under the conditions studied, even if there seems to be evidence of the formation of vortex structures for high couplings.

It is our intention in this contribution to summarize the essential features of the application of the inhomogeneous OZ approach to bulk Heisenberg magnetic fluids, both with ferromagnetic $[19,22]$ and antiferromagnetic $[11,27]$ interactions. The approach followed will be the one proposed by Lado and coworkers [19,22], although we will attempt to make connections with other formalisms where needed. The straightforward extension to deal with systems with three dimensional interactions constrained in surfaces will also be sketched and its application to dipolar ferrofluids illustrated. The next section will be devoted to a short presentation of the method. Then section III will focus on the specifics of ferro and antiferromagnetic spin fluids and finally in section IV we will briefly discuss the peculiarities of ferrofluid monolayers.

\section{The formalism}

In order to make the derivations more explicit, we specialize here on the Heisenberg spin fluid. In the next section the specific features of the dipolar case in two dimensions will be addressed.

The potential energy of a system composed of $\mathrm{N}$ hard spheres with embedded 
Heisenberg spins can be expressed

$$
U_{N}=\sum_{i<j}\left[u_{0}\left(r_{i j}\right)+u_{s s}\left(r_{i j}, \omega_{i}, \omega_{j}\right)\right]
$$

Here $u_{0}(r)$ is the hard sphere potential for spheres of diameter $\sigma$ and $u_{s s}\left(r, \omega_{1}, \omega_{2}\right)$ for $r>\sigma$ is the Heisenberg spin-spin interaction,

$$
\begin{aligned}
& u_{s s}\left(r, \omega_{1}, \omega_{2}\right)=-J(r) \hat{\mathbf{s}}_{1} \cdot \hat{\mathbf{s}}_{2}, \\
& \beta J(r)=K \frac{\mathrm{e}^{-\kappa(r / \sigma-1)}}{r / \sigma},
\end{aligned}
$$

where $\beta=1 / k_{\mathrm{B}} T$, with $k_{\mathrm{B}}$ Boltzmann's constant and $T$ the Kelvin temperature. The orientations $\omega=(\theta, \phi)$ of the unit spins $\hat{\mathbf{s}}$ are referred to a uniform field $\mathbf{B}_{0}$, which defines the $z$ direction. In the case of spontaneously broken symmetry in the zero field case, following Bogolubov [28], one resorts to the infinitesimal field which stabilizes the system [21]. In equation (3), the dimensionless coupling strength $K$ may be read as the inverse reduced temperature, $K=1 / T^{*}$, while $\kappa$ is a dimensionless range parameter. A positive $K$ will favour parallel alignments (ferromagnetic ordering) and a negative value will favour antiparallel alignment (antiferromagnetic ordering).

The key quantities needed for a complete magnetic and thermodynamic description of this system are the one-body and two-body density functions,

$$
\begin{aligned}
\rho^{(1)}(\mathbf{r}, \omega) & =\frac{\rho}{4 \pi} f(\omega), \\
\rho^{(2)}\left(\mathbf{r}, \omega, \mathbf{r}^{\prime}, \omega^{\prime}\right) & =\frac{\rho^{2}}{(4 \pi)^{2}} f(\omega) f\left(\omega^{\prime}\right) g\left(\left|\mathbf{r}-\mathbf{r}^{\prime}\right|, \omega, \omega^{\prime}\right),
\end{aligned}
$$

where $\rho=N / V$ is the density, $f(\omega)$ the one-body orientational distribution in the interacting fluid, and $g\left(r, \omega, \omega^{\prime}\right)$ the pair distribution function of the inhomogeneous spin system in an external magnetic field.

The basic equations that determine the distribution functions $f(\omega)$ and $g\left(r, \omega, \omega^{\prime}\right)$ are well known. The one-body density can be differentiated with respect to $x=\cos \theta$ to give

$$
\frac{\mathrm{d}}{\mathrm{d} x} \ln \left[\frac{f(\omega)}{f_{0}(\omega)}\right]=-\frac{\rho}{4 \pi} \int \mathrm{d} \mathbf{r} \mathrm{d} \omega^{\prime} f\left(\omega^{\prime}\right) g\left(r, \omega, \omega^{\prime}\right) \frac{\mathrm{d} \beta u_{s s}\left(r, \omega, \omega^{\prime}\right)}{\mathrm{d} x}
$$

the first member of a Kirkwood-Born-Green-Yvon hierarchy. Here $f_{0}(\omega)$ is the normalized one-body orientational distribution of a noninteracting spin system,

$$
f_{0}(\omega)=\frac{\mathrm{e}^{\beta \mu B_{0} \cos \theta}}{\sinh \left(\beta \mu B_{0}\right) / \beta \mu B_{0}},
$$

where $\mu$ is the spin dipole moment. Calculation of $f(\omega)$ from equation (6) requires knowing $g$. Alternatively, one can use Lovett's equation [18] which connects the one-body orientational distribution and the direct correlation function $c\left(r, \omega, \omega^{\prime}\right)$ via

$$
\nabla_{\omega} f(\omega)=\int c\left(r, \omega, \omega^{\prime}\right) \nabla_{\omega^{\prime}} f\left(\omega^{\prime}\right) \mathrm{d} \mathbf{r} \mathrm{d} \omega^{\prime}
$$


Both relations are exact, but obviously will lead to different results as long as the pair correlations functions are only approximated. In this regard, in classical liquid state theory, the pair distribution and direct correlation functions are obtained from the Ornstein-Zernike (OZ) equation and a closure relation respectively,

$$
\begin{aligned}
\gamma\left(r_{12}, \omega_{1}, \omega_{2}\right)= & \frac{\rho}{4 \pi} \int \mathrm{d} \mathbf{r}_{3} \mathrm{~d} \omega_{3} f\left(\omega_{3}\right)\left[\gamma\left(r_{13}, \omega_{1}, \omega_{3}\right)+c\left(r_{13}, \omega_{1}, \omega_{3}\right)\right] c\left(r_{32}, \omega_{3}, \omega_{2}\right), \\
c\left(r, \omega_{1}, \omega_{2}\right)= & \exp \left[-\beta u_{0}(r)-\beta u_{s s}\left(r, \omega_{1}, \omega_{2}\right)+\gamma\left(r, \omega_{1}, \omega_{2}\right)+b\left(r, \omega_{1}, \omega_{2}\right)\right] \\
& -1-\gamma\left(r, \omega_{1}, \omega_{2}\right)
\end{aligned}
$$

The first of these connects the indirect correlation function $\gamma=g-1-c$ with the direct correlation function $c$. The second, or closure, relation expresses $c$ back in terms of $\gamma$ and the model's pair interactions. This relation must be supplemented with an approximation for $b$, the so-called bridge function. This can be approximated in various ways giving rise to a variety of closures, like the reference-hypernetted chain (RHNC) closure [29] or the reference version of the Zerah-Hansen (RZH) closure [30]; for $r>\sigma$, these are

$$
\begin{aligned}
& c^{\mathrm{RHNC}}\left(r, \omega_{1}, \omega_{2}\right)= \exp \left[-\beta u_{s s}\left(r, \omega_{1}, \omega_{2}\right)+\gamma\left(r, \omega_{1}, \omega_{2}\right)+b_{\mathrm{HS}}\left(r ; \sigma_{\mathrm{HS}}\right)\right] \\
&-1-\gamma\left(r, \omega_{1}, \omega_{2}\right), \\
& c^{\mathrm{RZH}}\left(r, \omega_{1}, \omega_{2}\right)=\left\{\begin{aligned}
\exp \left(m(r)\left[-\beta u_{s s}\left(r, \omega_{1}, \omega_{2}\right)+\gamma\left(r, \omega_{1}, \omega_{2}\right)+b_{\mathrm{HS}}(r ; \sigma)\right]\right) \\
\quad-1\} / m(r)-\gamma\left(r, \omega_{1}, \omega_{2}\right) .
\end{aligned}\right.
\end{aligned}
$$

In equation(11) $b_{\mathrm{HS}}\left(r ; \sigma_{\mathrm{HS}}\right)$ represents the bridge function of a fluid of hard spheres of diameter $\sigma_{\mathrm{HS}}$. For the potential models considered in this contribution we have simply used $\sigma_{\mathrm{HS}}=\sigma$. The RZH closure features a mixing function $m(r)=1-$ $\exp (-\alpha r)$ with a parameter $\alpha$ that is fixed by requiring consistency between the virial and compressibility bulk moduli [30]. In the limiting case of $\alpha \rightarrow 0$, the RZH closure simply reproduces the MSA closure.

The usual approach for orientation-dependent functions such as $\gamma\left(r, \omega_{1}, \omega_{2}\right)$ is to expand in spherical harmonics as both Sokolovska [17] and Perera [23] did, leading to the appearance of matrix terms of the form

$$
R^{k l \mu}=\int \rho(\omega) Y_{k \mu}(\omega) Y_{l \bar{\mu}}(\omega) \mathrm{d}(\omega)
$$

(or $A^{k l \mu}$ in the notation of Klapp and Patey [25] and $Y^{k l \mu}$ in Sokolovska's work [17]) once the $\mathrm{OZ}$ equation is decoupled into its components. These terms appear both in the equation itself and in the expressions to evaluate thermodynamic properties. Here we will instead expand in modified spherical harmonics,

$$
\begin{aligned}
\gamma\left(r, \omega_{1}, \omega_{2}\right) & =4 \pi \sum_{l_{1}, l_{2}, m} \gamma_{l_{1} l_{2} m}(r) \mathcal{Y}_{l_{1} m}\left(\omega_{1}\right) \mathcal{Y}_{l_{2} \bar{m}}\left(\omega_{2}\right) \\
\mathcal{Y}_{l m}(\omega) & =\frac{1}{\sqrt{4 \pi}}(-1)^{m} \mathrm{e}^{\mathrm{i} m \phi} \mathcal{P}_{l m}(\cos \theta)
\end{aligned}
$$


The modified Legendre functions $\mathcal{P}_{l m}(\cos \theta)$ are explicitly constructed using the Gram-Schmidt method with the orthonormality condition

$$
\frac{1}{2} \int_{-1}^{1} \mathrm{~d} x f(x) \mathcal{P}_{l m}(x) \mathcal{P}_{l^{\prime} m}(x)=\delta_{l l^{\prime}}
$$

where $f(\cos \theta)$ is the one-body distribution of the fluid. Obviously this construction eliminates the $R^{k l \mu}$ matrices from all subsequent expressions and as will be seen yields equations for the coefficients completely analogous to those of the isotropic integral equations.

Now, the OZ equation (9) deconvoluted by Fourier transformation and with the pair functions expanded as in (14) becomes

$$
\tilde{\gamma}_{l_{1} l_{2} m}(k)=(-1)^{m} \rho \sum_{l_{3}}\left[\tilde{\gamma}_{l_{1} l_{3} m}(k)+\tilde{c}_{l_{1} l_{3} m}(k)\right] \tilde{c}_{l_{3} l_{2} m}(k) .
$$

The significant feature here is that this OZ equation for an inhomogeneous fluid in an external field is now identical to that of an ordinary homogeneous fluid and so, along with a closure equation, can be solved for $g\left(r, \omega_{1}, \omega_{2}\right)$ with the same familiar algorithms already used for homogeneous systems [31].

This solution for $g\left(r, \omega_{1}, \omega_{2}\right)$ is obtained using the polynomials $\mathcal{P}_{l m}(x)$ generated with the current $f(x)$. We now return to equation (6) and update the one-body distribution. In expanded form, this BG equation is

$$
\begin{aligned}
\frac{\mathrm{d}}{\mathrm{d} x} \ln \left[\frac{f(x)}{f_{0}(x)}\right] & =\sum_{l_{1}, l_{2}, m} \xi_{l_{1} l_{2} m} \mathcal{P}_{l_{1} m}(x) \frac{\mathrm{d} \mathcal{P}_{l_{2} m}(x)}{\mathrm{d} x} \\
\xi_{l_{1} l_{2} m} & =-\rho \int \mathrm{d} \mathbf{r} \sum_{l_{3}} g_{l_{1} l_{3} m}(r) u_{l_{3} l_{2} \bar{m}}(r),
\end{aligned}
$$

where the $u_{l_{1} l_{2} m}(r)$ are the (known) coefficients of the spin-spin interaction $u_{s s}\left(r, \omega_{1}, \omega_{2}\right)$, so that finally

$$
\ln f(x)=\ln f_{0}(x)+\sum_{l=0}^{\infty} a_{l} \mathcal{P}_{l 0}(x) .
$$

Here $a_{l}$ for $l>0$ is determined by numerical (Gaussian) integration of equation (16) and $a_{0}$ by normalization.

The iterations for $f(\omega)$ and $g\left(r, \omega_{1}, \omega_{2}\right)$ are continued until both functions are selfconsistently determined. We can now compute the complete magnetic and thermodynamic properties of a Heisenberg spin fluid in a uniform magnetic field $\mathbf{B}_{0}=B_{0} \hat{\mathbf{k}}$ or in the ferromagnetic phase. In particular, we find for the longitudinal and transverse magnetic susceptibilities the following:

$$
\begin{aligned}
\chi_{z z} / \rho \beta \mu^{2}= & \sigma_{x}^{2}\left[1+\rho \tilde{h}_{110}(0)\right]+\langle x\rangle \sigma_{x}\left[\rho \tilde{h}_{100}(0)+\rho \tilde{h}_{010}(0)\right] \\
& +\left\langle x^{2}\right\rangle\left[1+\rho \tilde{h}_{000}(0)\right] \\
\chi_{y y} / \rho \beta \mu^{2}= & \frac{1}{2}\left(1-\left\langle x^{2}\right\rangle\right)\left[1-\rho \tilde{h}_{111}(0)\right]
\end{aligned}
$$


where $\sigma_{x}^{2} \equiv\left\langle x^{2}\right\rangle-\langle x\rangle^{2}$ for $x=\cos \theta$. In connection with the susceptibilities there is an important aspect that deserves to be born in mind. It is well known that the spontaneous symmetry breaking associated with the ferromagnetic transition gives rise to transverse fluctuations, known as Goldstone modes, which reflect in a divergence of the transverse magnetic susceptibility. It has been proved that Lovett's equation automatically reproduces the presence of Goldstone modes in orientationally ordered phases $[17,23,21]$, but this is not evident when the BG equation is used. In fact, we have found that the MSA transverse magnetic susceptibility remains finite in the ferromagnetic phases at zero field, whereas the RHNC equation breaks down at small but finite fields, signalling the presence of a divergence. Therefore, we have taken advantage of these features to obtain zero field results from the RZH approximation. Being a hybrid between MSA and RHNC one can always obtain a solution for $\alpha=0$ and then increase its value until the divergence is reached. We have found that the values in the immediate vicinity of the divergence agree extremely well with zero-field computer simulation estimates. A more elegant approach would certainly imply an analytic treatment of the divergence following Perera [23]. On the other hand, one might perform the calculations at small field as Klapp and Patey [25] suggested. Sokolovska [17] uses Baxter's method to analytically solve the MSA and consequently her treatment is not affected by the divergence.

\section{Bulk Heisenberg fluids: ferromagnetic and antiferromagnet- ic ordering}

Here we present a set of calculations is for $\kappa=1, \rho \sigma^{3}=0.7, K=1 / T^{*}=0$ to 0.5 , and two values of the external field, $\beta \mu B_{0}=1$ and 0 , using the RZH closure. We find for these cases that the one-body orientational distribution function $f(x)$ continues to be well described by the functional form of $f_{0}(x)$, but with an effective field $B$,

$$
f(x)=\frac{\mathrm{e}^{\beta \mu B x}}{\sinh (\beta \mu B) / \beta \mu B} .
$$

In the limit $B_{0} \rightarrow 0$, the effective field $B$ is zero for $K<K_{\mathrm{c}}$ and finite for $K>K_{\mathrm{c}}$, where $K_{\mathrm{c}}=1 / T_{\mathrm{c}}^{*}$ is then the computed Curie point.

For $K>K_{\mathrm{c}}$, there is a singularity when $B_{0} \rightarrow 0$ due to the presence of the Goldstone modes. In fact, the $B_{0}=0$ line corresponds in the $B_{0}-M$ plane (where $M$ is the magnetization per particle) to the spinodal line that indicates the equilibrium between phases with positive and negative magnetization. Consequently, the transverse susceptibility $\chi_{y y}$ (but not the longitudinal component $\chi_{z z}$ ) will diverge as $B_{0} \rightarrow 0$, reflecting the negligible cost of rotating an ordered sample in the absence of an external field.

Using Monte Carlo simulation, Nijmeijer and Weis [13] found that the paramagnetic-ferromagnetic transition for $\rho \sigma^{3}=0.7$ with the truncated potential occurs at $K_{\mathrm{c}}=0.264 \pm 0.001$. Our calculation in the paramagnetic phase and zero field limit yields a divergence in $\chi_{z z}$ at $K_{\mathrm{c}}{ }^{1}=0.2645$, in excellent agreement with the simulation value. 


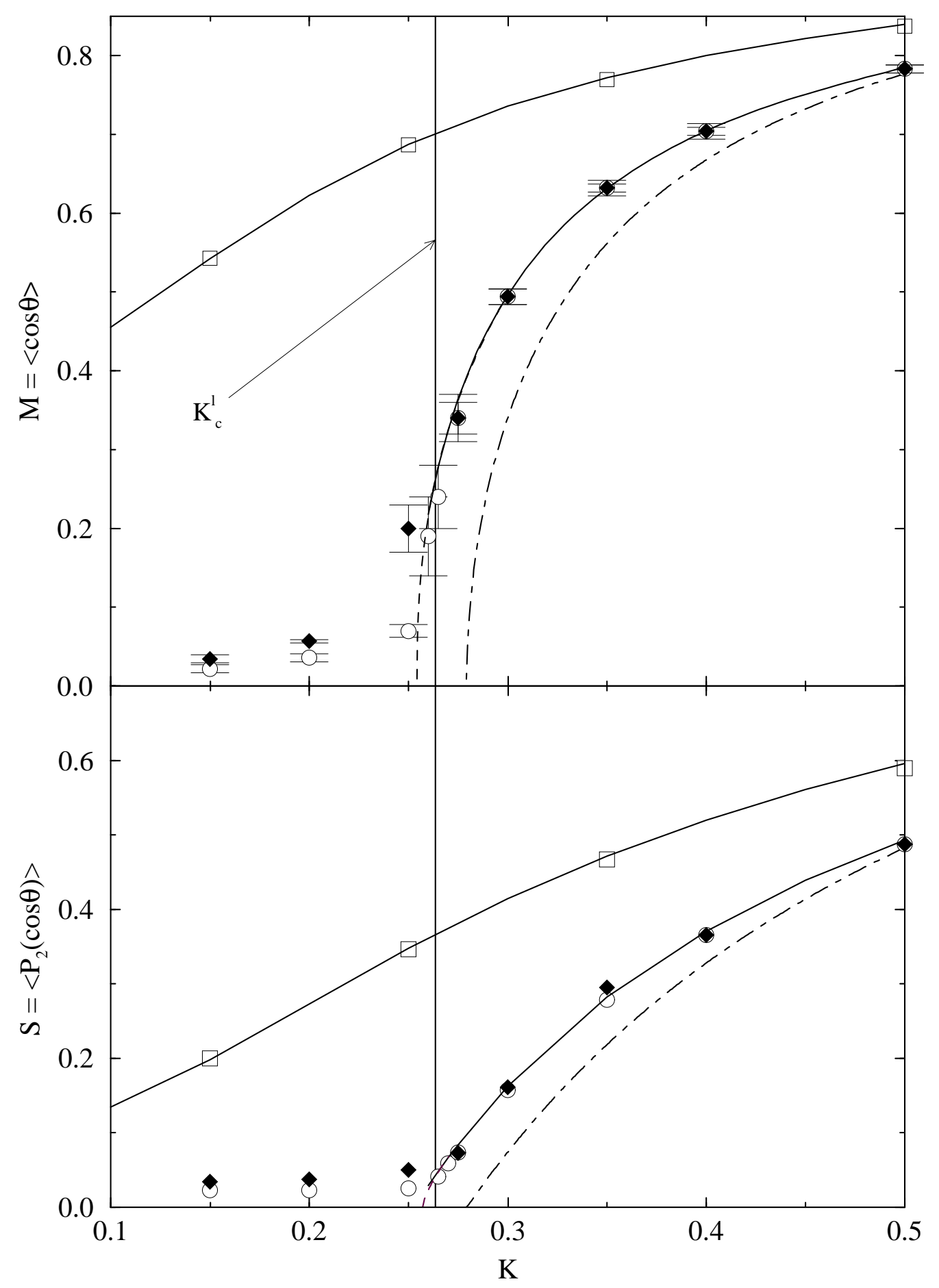

Figure 1. Magnetization per particle $M$ and second order parameter $S$ as functions of inverse temperature $K=1 / T^{*}$ obtained from MC simulation (squares for $\beta \mu B_{0}=1$, and white circles [2048 particle sample] and black diamonds [1372 particle sample] for $\beta \mu B_{0}=0$ ) and the RZH integral equation (solid lines). The dashed lines correspond to a power law fit to the RZH data just above $K_{\mathrm{c}}^{\mathrm{u}}$. The dash-dot curves represent density functional theory estimates. 


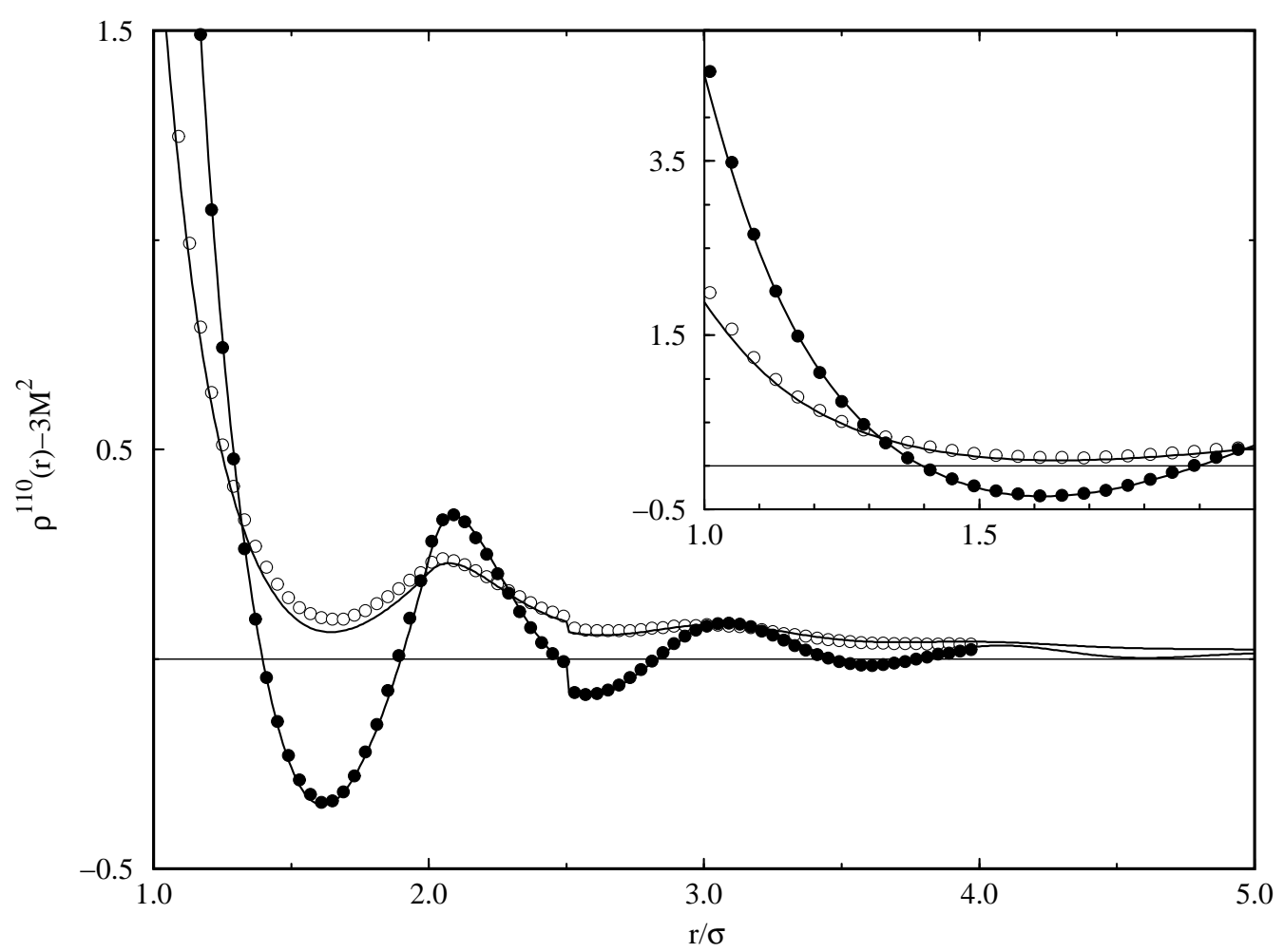

Figure 2. The $\rho_{(2)}^{110}(r)$ angular correlation function from simulation (open circles) and the RZH integral equation (solid lines) for $K=0.5$ with and without an external magnetic field. The long-range limiting value $3 M^{2}$ has been subtracted from both theory and simulation results to ease the comparison between the two temperatures. The discontinuity at $r=2.5 \sigma$ is due to the potential truncation.

In figure 1 we show the values of the magnetization per particle $M \equiv M_{z}=$ $\langle\cos \theta\rangle$ and the second order parameter $S=\left\langle P_{2}(\cos \theta)\right\rangle$ obtained from the theory and from a standard NVT Monte Carlo simulation using 864 (for $B_{0}=1$ ), 1372 and 2048 particles (for $B_{0}=0$ ), and averages over $4 \times 10^{4}$ configurations. Size effects are noticeable for $B_{0}=0$ close to the critical point. In the vicinity of the critical temperature, one encounters convergence difficulties in solving the integral equation as spin-spin correlations become long ranged. Both $\chi_{z z}$ and $\chi_{y y}$ in equations (19) and (20) diverge, with $\chi_{z z}$ exhibiting the characteristic $\lambda$-type divergence of second-order transitions.

In order to get an estimate of the critical inverse temperature from the orderedphase results, we fit the RZH magnetization in the vicinity of the critical temperature $(K<0.3)$ to a power law,

$$
M=a\left(K-K_{\mathrm{c}}^{\mathrm{u}}\right)^{\beta},
$$

which leads to $a=1.694, \beta=0.397$, and $K_{\mathrm{c}}^{\mathrm{u}}=0.254$. The fitted value of $\beta$ is close to the value $\beta=0.387$ reported by Nijmeijer and Weis [13] and to the critical exponent of the 3D lattice Heisenberg model, $\beta=0.362 \pm 0.004$ [32] and $\beta=0.3639 \pm 0.0035$ [33]. However, the available data from the integral equation magnetization are too far away from $K_{\mathrm{c}}$ to draw here any conclusion with confidence and this agreement 
might simply be fortuitous. Nevertheless, a similar fit carried out on the secondorder parameter $S$ produces the same estimate of $K_{\mathrm{c}}^{\mathrm{u}}$ and so we might conclude that this is the theoretical estimate of the critical inverse temperature derived from the ordered-phase data. The agreement between the critical point estimates $K_{\mathrm{c}}{ }^{1}=0.2645$ obtained in the isotropic phase and $K_{\mathrm{c}}^{\mathrm{u}}=0.254$ obtained in the ordered phase can be regarded as good.

In figure 2 we plot one of the averaged angular correlations most representative of the orientational ordering and which in the isotropic case reduces to the standard $\mathrm{h}^{110}$ coefficient of the expansion of the pair distribution function in rotational invariants. In the anisotropic case, the corresponding average can be related to the coefficients of the expansion in special orthogonal polynomials by means of

$$
\begin{aligned}
\rho_{(2)}^{110}(r) / \rho^{2} & \equiv 3\left\langle\rho^{(2)}(12) / \rho^{2} \phi^{110}(12)\right\rangle_{\omega_{1} \omega_{2}} \\
& =3\left[\langle x\rangle^{2} g_{000}(r)+2\langle x\rangle \sigma_{x} g^{010}(r)+\sigma_{x}^{2} g_{110}-\left(1-\left\langle x^{2}\right\rangle\right) g_{111}(r)\right] .
\end{aligned}
$$

We have found that both the structural and thermodynamics properties are correctly predicted by the RZH equation in zero field. For finite fields the RHNC equation is slightly better and has the advantage that properties like the free energy can be directly evaluated from the correlation functions [22]. We are thus in a position to produce estimates for the phase diagram using the RZH equation at zero field via thermodynamic integration, and applying a double tangent construction to the RHNC free energies at finite fields. We also consider here a fully aligned spin system, i.e., a spin fluid under the action of an infinitely strong external field. The phase equilibria results are presented in figure 3, along with GEMC data. Comparing the infinite field (i.e. pure Yukawa) and the zero field results in this figure, one notes that the equilibrium densities are only slightly affected by the external field. We have therefore chosen a relatively large field, $\beta \mu B_{0}=16$, to perform an additional set of calculations at nonzero but finite field. The results obtained from the RHNC approximation, seen in figure 3, although relatively good, are somewhat worse than those obtained for the pure Yukawa; this is a direct consequence of the neglect of the optimization condition for finite field. The situation is slightly worse using the RZH equation in the zero field case. Here the use of thermodynamic integration based on the energy route, which yields extremely good thermodynamics, is too time consuming, since it has to be performed for every density needed to map the isotherms required for the double tangent construction. Consequently, we have used the virial route starting from low density results, which is somewhat poorer, since RZH virial pressures are not as accurate as the corresponding internal energies. Therefore, we have only calculated two equilibrium points (each implies one hundred integral equation solutions) which are shown in the lower part of figure 3.

Finally, both theory and simulation show that the effect of an external field on the spin system (and presumably also on dipolar fluids [34]) is a considerable increase in the critical temperature, while equilibrium densities are not significantly affected; i.e., external fields tend to stabilize the liquid phase.

Now, if the sign of the coupling constant $K$ in equation(3) is changed the system will now exhibit antiferromagnetic behaviour. Already in [11] it was found that 


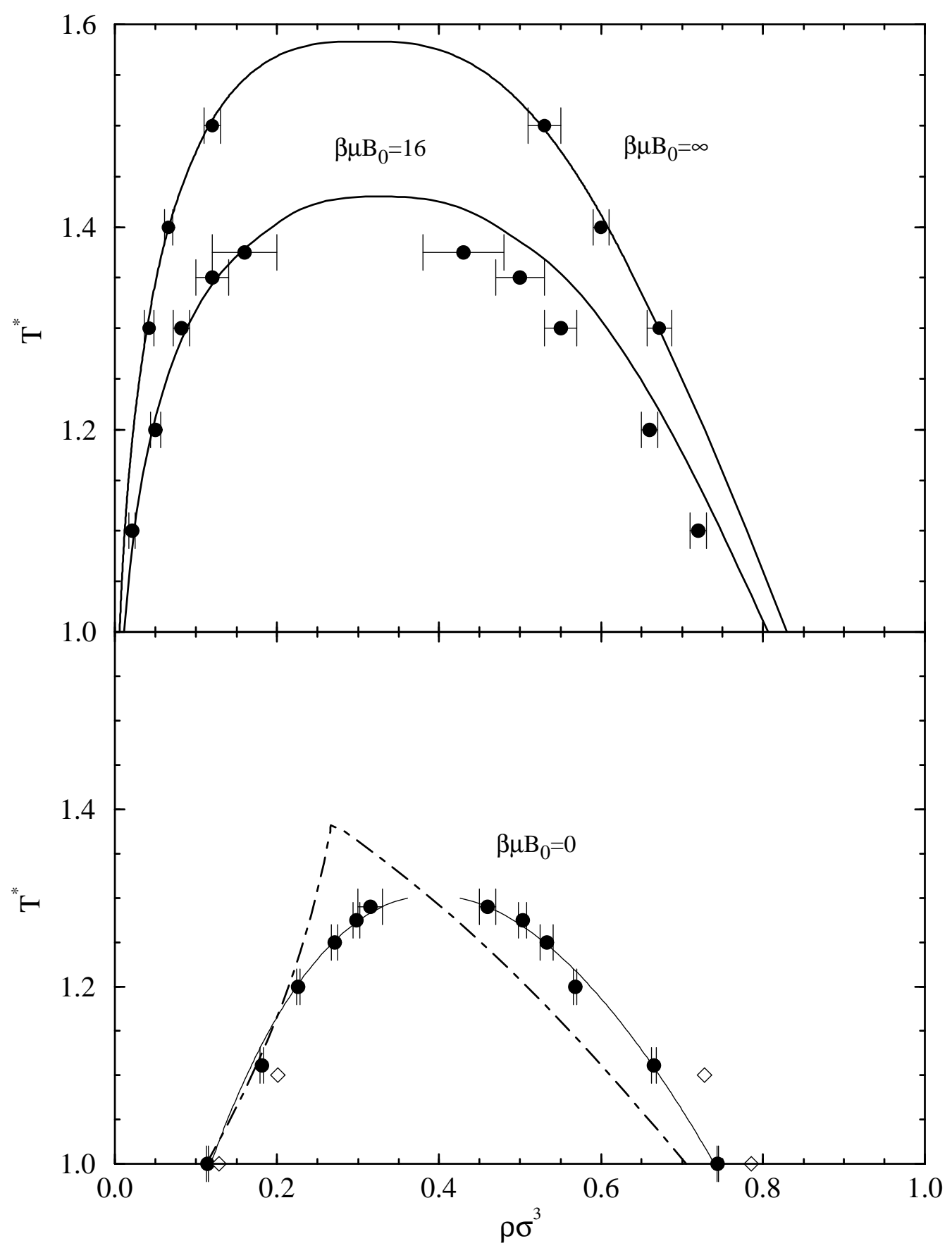

Figure 3. Phase diagram of the Heisenberg spin fluid in the presence of an external field (upper figure) and at zero field (lower figure). Simulation data are shown as black circles and RHNC results as solid lines. In the lower figure, the dash-dot line indicates density functional theory results taken from [12] while white diamonds represent two equilibrium points obtained from thermodynamic integration of the RZH results. 
Table 1. Computed order parameter and thermodynamic properties of the antiferromagnetic Heisenberg spin fluid for $\rho \sigma^{3}=0.8$ and $\kappa=6$.

\begin{tabular}{ccccccc}
$K$ & $\left\langle P_{2}\left(\cos \theta_{12}\right\rangle\right.$ & $a$ & $\beta P / \rho$ & \multicolumn{2}{c}{$\beta U / N$} \\
\hline & RZH & MC & RZH & RZH & RZH & MC \\
\hline-4.0 & 0 & - & 0 & 3.612 & -2.834 & - \\
-5.0 & 0 & - & 0 & 2.034 & -4.144 & - \\
-5.5 & 0 & - & 0 & 0.625 & -4.650 & - \\
-5.7 & 0 & - & 0 & 0.135 & -4.910 & - \\
-5.8 & 0.502 & - & -3.50 & -0.303 & -5.894 & - \\
-5.9 & 0.553 & - & -3.96 & -0.685 & -6.181 & - \\
-6.0 & 0.581 & 0.65 & -4.24 & -0.835 & -6.470 & -7.8 \\
-7.0 & 0.745 & - & -6.66 & -3.170 & -8.942 & - \\
-8.0 & 0.811 & 0.81 & -8.65 & -5.589 & -11.232 & -12.7
\end{tabular}

the paramagnetic-antiferromagnetic transition occurs at much lower temperatures (higher couplings) than the the ferromagnetic transition. This was particularly evident given the difficulties encountered by the simulation to attain antiferromagnetic ordering, which apparently takes much longer to build. This is connected with the fact that, whereas the propagation of ferromagnetic ordering is energetically favourable (all layers of parallel particles tend to lower the energy of the system) in the case of antiferromagnetic interactions successive layers of ordered spins have opposing contributions, i.e., first neighbours are antiparallel (lowering the energy) whereas second neighbours being parallel tend to increase the energy, and so on. The conditions for the formation of antiferromagnetic phases are then too severe to be easily handled by the simulation procedures and hinder the convergence of integral equations. However, we have found that decreasing the range of the interaction translates into a considerable lowering of the critical $K_{\mathrm{c}}$. Thus, whereas isotropic RHNC calculations indicate that, at $\rho \sigma^{3}=0.8$, the transition occurs for $K_{\mathrm{c}} \approx 10$ for a screening factor $\kappa=1$, changing this to $\kappa=6$ (which implies that practically only nearest neighbours are involved in the interaction) reduces the critical coupling to $K_{\mathrm{c}} \approx 4.3$. Nonetheless, these conditions also pose important problems to the simulation procedures, in particular since the low temperature combined with the short range translates into a certain 'sticky' character that induces a considerable amount of clustering. Thermodynamics and order parameters obtained for this model using RZH and simulation for various states are collected in table I. These results are still very preliminary but indicate that despite the difficulties the method can still be applied to antiferromagnetic systems. It turns out that the one particle distribution function in these systems follows a functional form

$$
f(x)=\frac{\mathrm{e}^{a x^{2}}}{2 e D(1)},
$$

where $D(t)$ is a Dawson's integral and $a>0$. In the relative large region around 
the antiferromagnetic transition the integral equation experiments considerable convergence difficulties, and we have found that a simple density functional theory of the type developed in [12] predicts this transition to be slightly first order. All this clearly indicates that the antiferromagnetic system deserves further study.

\section{Ferrofluid monolayers}

The interaction energy for a configuration of $N$ sphere centers $\mathbf{r}_{j}$ with embedded three-dimensional magnetic moments $\boldsymbol{\mu}_{j}$ in a plane area $A$ is

$$
U=\sum_{i<j} u_{0}\left(r_{i j}\right)+\sum_{i<j} u_{d d}\left(\mathbf{r}_{i j}, \omega_{i}, \omega_{j}\right)-\sum_{j} \boldsymbol{\mu}_{j} \cdot \mathbf{B}_{0},
$$

where

$$
u_{d d}\left(\mathbf{r}_{12}, \omega_{1}, \omega_{2}\right)=-\frac{\mu^{2}}{r_{12}^{3}}\left[3\left(\hat{\mathbf{r}}_{12} \cdot \hat{\boldsymbol{\mu}}_{1}\right)\left(\hat{\mathbf{r}}_{12} \cdot \hat{\boldsymbol{\mu}}_{2}\right)-\hat{\boldsymbol{\mu}}_{1} \cdot \hat{\boldsymbol{\mu}}_{2}\right]
$$

is the dipole-dipole potential, and $\mathbf{B}_{0}$ a uniform magnetic field perpendicular to the plane. Even in the absence of the magnetic field, the planar arrangement of the point dipoles produces anisotropy.

With the particles laying on a plane and placing the $x$-axis along the intermolecular axis, (26) reduces to

$$
\begin{aligned}
u_{d d}\left(r_{12}, \theta_{1}, \theta_{2}, \phi\right)=-\frac{\mu^{2}}{r^{3}} & {\left[\frac{3}{2} \sin \theta_{1} \sin \theta_{2} \cos \left(\phi_{1}+\phi_{2}\right)\right.} \\
& \left.+\frac{1}{2} \sin \theta_{1} \sin \theta_{2} \cos \left(\phi_{1}-\phi_{2}\right)-\cos \theta_{1} \theta_{2}\right],
\end{aligned}
$$

where $\theta_{i}$ denotes the angle formed by dipole $i$ with the plane normal and $\phi_{i}$ is the azimuthal angle. Clearly one can consider this as purely one dimensional problem with particles carrying an additional internal degree of freedom quantified by $\theta_{i}$. We can then simply make use of the expressions introduced in the previous section with minor changes to reflect the different symmetry of the problem and the reduction in dimensionality. Thus, first we will have to expand the correlation functions in our new set or orthogonal polynomials as,

$$
\gamma\left(\mathbf{r}, \omega_{1}, \omega_{2}\right)=4 \pi \sum_{l_{1}, l_{2}, m_{1}, m_{2}} \gamma_{l_{1} l_{2}}^{m_{1} m_{2}}(r) \mathcal{Y}_{l_{1} m_{1}}\left(\omega_{1}\right) \mathcal{Y}_{l_{2} \bar{m}_{2}}\left(\omega_{2}\right)
$$

Similarly, Fourier transforms, which will be used to deconvolute the OZ equation, are also conveniently expanded with the $x$ axis along the planar vector $\mathbf{k}$. A twodimensional Fourier transform of the correlation functions is then evaluated as follows. Choose the $x$ axis along $\mathbf{k}$. Then using (28) we have

$$
\begin{aligned}
\tilde{\gamma}(12) & =\int \mathrm{d} \mathbf{r} \gamma(12) \mathrm{e}^{\mathrm{i} k r \cos \varphi_{r}} \\
& =4 \pi \sum_{l_{1}, l_{2}, m_{1}, m_{2}} \int_{0}^{\infty} \mathrm{d} r r \gamma_{l_{1} l_{2}}^{m_{1} m_{2}}(r)
\end{aligned}
$$




$$
\begin{gathered}
\quad \times \int_{0}^{2 \pi} \mathrm{d} \varphi_{r} \mathrm{e}^{\mathrm{i} k r \cos \varphi_{r}} \mathrm{e}^{-i\left(m_{1}-m_{2}\right) \varphi_{r}} \mathcal{Y}_{l_{1} m_{1}}\left(\omega_{1}\right) \mathcal{Y}_{l_{2} \bar{m}_{2}}\left(\omega_{2}\right) \\
=4 \pi \sum_{l_{1}, l_{2}, m_{1}, m_{2}} \tilde{\gamma}_{l_{1} l_{2}}^{m_{1} m_{2}}(k) \mathcal{Y}_{l_{1} m_{1}}\left(\omega_{1}\right) \mathcal{Y}_{l_{2} \bar{m}_{2}}\left(\omega_{2}\right),
\end{gathered}
$$

where

$$
\tilde{\gamma}_{l_{1} l_{2}}^{m_{1} m_{2}}(k)=2 \pi \mathrm{i}^{m_{1}-m_{2}} \int_{0}^{\infty} \mathrm{d} r r \gamma_{l_{1} l_{2}}^{m_{1} m_{2}}(r) J_{\left|m_{1}-m_{2}\right|}(k r) .
$$

Here, $J_{m}(x)$ is the Bessel function of order $m$ generated by the integral over $\varphi_{r}$. Similarly, an inverse transform is

$$
\gamma_{l_{1} l_{2}}^{m_{1} m_{2}}(r)=\frac{1}{2 \pi \mathrm{i}^{m_{1}-m_{2}}} \int_{0}^{\infty} \mathrm{d} k k \tilde{\gamma}_{l_{1} l_{2}}^{m_{1} m_{2}}(k) J_{\left|m_{1}-m_{2}\right|}(k r) .
$$

It follows from the circular symmetry of the system that $\left|m_{1}-m_{2}\right|$ must be an even integer.

We find by calculation that in this case the angular distribution function can be cast into the form

$$
f(x)=\frac{1}{C} \exp \left(\beta \mu^{2} K x^{2}+\beta \mu B x\right),
$$

In equation (32), $K$ and $B$ are effective values of the magnetic surface anisotropy and external magnetic field, respectively, while the normalization constant $C$ is given by

$$
C=\frac{1}{4} \sqrt{\frac{\pi}{a}} \mathrm{e}^{b^{2} / 4 a}\left[\operatorname{erf}\left(\frac{2 a-b}{2 \sqrt{a}}\right)+\operatorname{erf}\left(\frac{2 a+b}{2 \sqrt{a}}\right)\right] .
$$

where $a=\beta \mu^{2} K$ and $b=\beta \mu B$. In contrast with the antiferromagnetic fluid, here $a<0$ reflecting the symmetry of the angular distribution at zero field.

A comparison between our Monte Carlo data for $f(x)$ and the results from the RHNC, integral equation is displayed in figure 4 for various external fields. Given that the magnitude of common magnetic dipoles is appreciably smaller than that of their electric counterparts, we have only considered a relatively moderate reduced dipole, $\mu^{*}=\left(\beta \mu^{2} / \sigma^{3}\right)^{1 / 2}=1$. The agreement seen in the figure between the integral equation and the simulation results is excellent. The most noticeable feature observed is the breaking of the symmetry of the distribution with respect to the plane $(x=\cos \theta=0)$ due to the external field. We have found that obviously the response of the dipoles to the field is weaker at higher densities. This effect of density is readily understandable, since the dipole-dipole interaction favouring head-to-tail alignments increases the tendency of the dipoles to remain in-plane. The same effect can also be seen in the zero-field truncated Gaussian distribution of figure 4, which becomes progressively more sharply peaked about $x=0$ as the density increases, approaching a fully coplanar distribution. A similar effect can be induced by an increase of the dipole moment.

As to the pair structure of the system, in dipolar fluids, in addition to the centerto-center pair distribution function $g^{000}(r) \equiv g_{00}^{00}(r)$, the most significant angular correlations are represented by the ensemble shell averages - projections of the normalized two particle density $\rho^{(2)}(12) / \rho^{2}$ - of the standard rotational invariants 


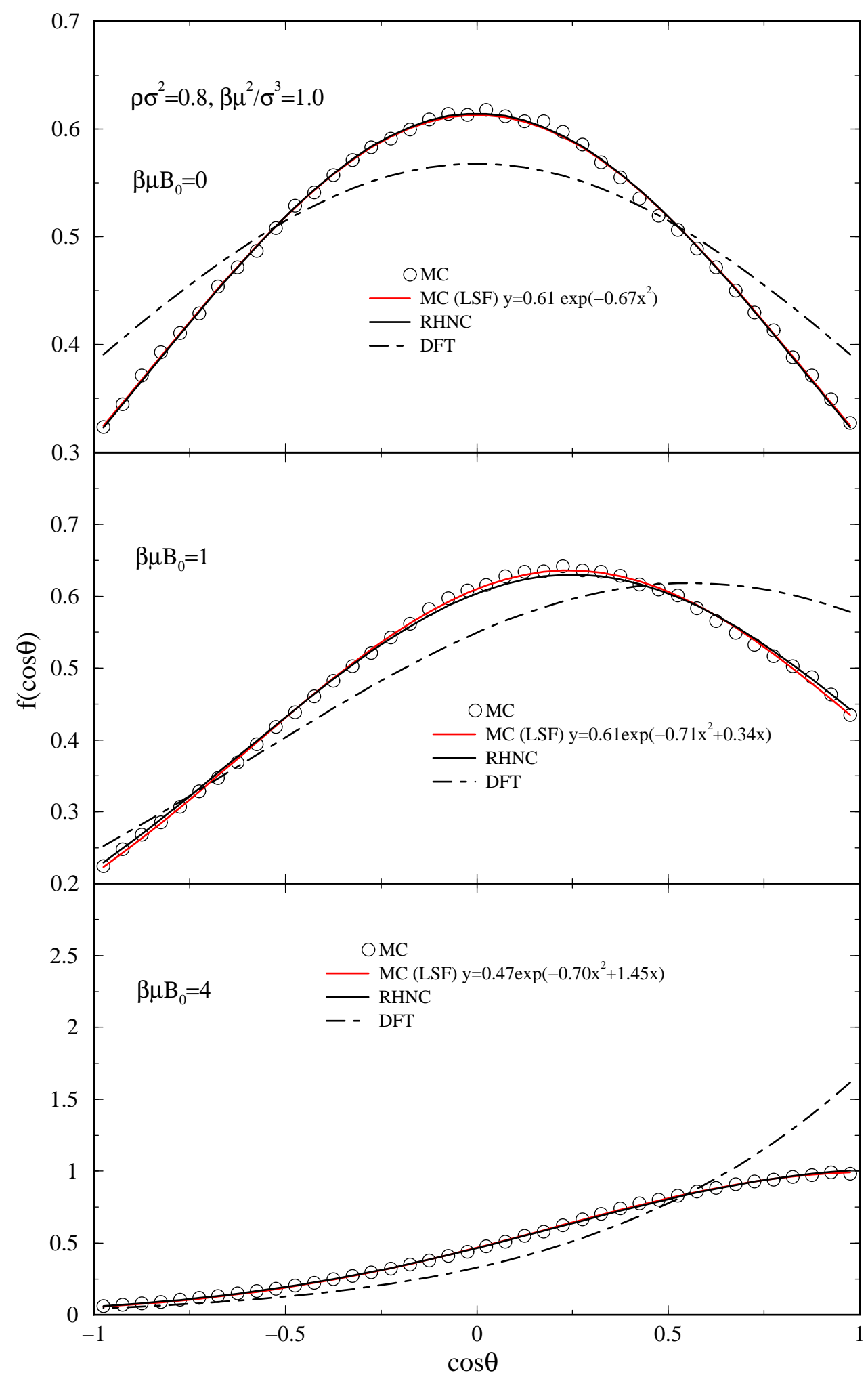

Figure 4. Angular distribution function $f(\cos \theta)$ in a ferrofluid monolayer of dipolar hard spheres at $\rho \sigma^{2}=0.8$ and various external fields. Open circles denote MC data and solid line RHNC results. The dotted line tracking the MC points is a least squares fit (LSF) whose coefficients are shown in the figure. 
$\phi^{110}=\hat{\boldsymbol{\mu}}_{1} \cdot \hat{\boldsymbol{\mu}}_{2}$ and $\boldsymbol{\phi}^{112}=3\left(\hat{\boldsymbol{\mu}}_{1} \cdot \hat{\mathbf{r}}\right)\left(\hat{\boldsymbol{\mu}}_{2} \cdot \hat{\mathbf{r}}\right)-\hat{\boldsymbol{\mu}}_{1} \cdot \hat{\boldsymbol{\mu}}_{2}$, which in the isotropic case reduce to the coefficients $h^{110}(r)$ and $h^{112}(r)$. These quantities are connected respectively to the relative orientation of a pair of dipoles at a given separation and to the contribution of a given spherical shell to the dipolar excess energy. In terms of the calculated coefficients $g_{l_{1} l_{2}}^{m_{1} m_{2}}(r)$ of this work, the relevant averaged quantities are given by

$$
\begin{aligned}
\rho_{(2)}^{110}(r) / \rho^{2} & \equiv 3\left\langle\rho^{(2)}(12) / \rho^{2} \phi^{110}(12)\right\rangle_{\omega_{1} \omega_{2}} \\
& =3\left[\langle x\rangle^{2} g_{00}^{00}(r)+2\langle x\rangle \sigma_{x} g_{01}^{00}(r)+\sigma_{x}^{2} g_{11}^{00}-\left(1-\left\langle x^{2}\right\rangle\right) g_{11}^{11}(r)\right]
\end{aligned}
$$

and

$$
\begin{aligned}
\rho_{(2)}^{112}(r) / \rho^{2} \equiv & \frac{3}{2}\left\langle\rho^{(2)}(12) / \rho^{2} \phi^{112}(12)\right\rangle_{\omega_{1} \omega_{2}} \\
= & -\frac{3}{2}\left[\langle x\rangle^{2} g_{00}^{00}(r)+2\langle x\rangle \sigma_{x} g_{01}^{00}(r)+\sigma_{x}^{2} g_{11}^{00}(r)+\frac{1}{2}\left(1-\left\langle x^{2}\right\rangle\right) g_{11}^{11}(r)\right. \\
& \left.\quad-\frac{3}{2}\left(1-\left\langle x^{2}\right\rangle\right) g_{11}^{1-1}(r)\right] .
\end{aligned}
$$

The correlation functions obtained from the RHNC integral equation are compared with MC simulation data in figures 5-6 for various densities and external fields. It can be appreciated that the optimized RHNC integral equation provides an excellent description of the microscopic structure of the dipolar fluid, with and without external field, except in the case of $\rho \sigma^{2}=0.8$. Here the integral equation renders a much more pronounced orientational structure while the spatial ordering is exactly reproduced. This discrepancy will be further discussed below when analyzing the thermodynamic properties. The behaviour at large separations is well reproduced, including the crossover of $\rho_{(2)}^{110}(r)$ and $\rho_{2}^{112}(r)$. We have also found that the contact values of the correlation functions decrease slightly as the field is augmented; this is due to the fact that the out-of-plane alignment induced by the field introduces repulsive dipole-dipole interactions. This effect is obviously less significant at low densities.

It is worth noting that we have encountered convergence problems in the RHNC solutions for reduced densities above 0.8. As can be seen in figure 7, this corresponds to a region where the transverse (i.e., in-plane) susceptibility starts to rise appreciably and where the orientational order (see figure 6) predicted by the integral equation is more pronounced than what is actually found in the simulation. This orientational structure disagreement is in consonance with the discrepancies in the behaviour of the transverse susceptibility depicted in figure 7 . Thus, whereas the increase in the theoretical susceptibility and the difficulties in convergence seem to indicate that the system might be close to some sort of in-plane order-disorder transition, the simulation predicts no anomalous behaviour for these high density states. Nonetheless, at somewhat higher dipole moment the simulation results start to show a clear head-to-tail in-plane alignment of the dipoles with the formation of vortex structures, as can be appreciated in figure 8. It thus might happen that the integral equation underestimates the value of the transition dipole moment. Alternatively, 


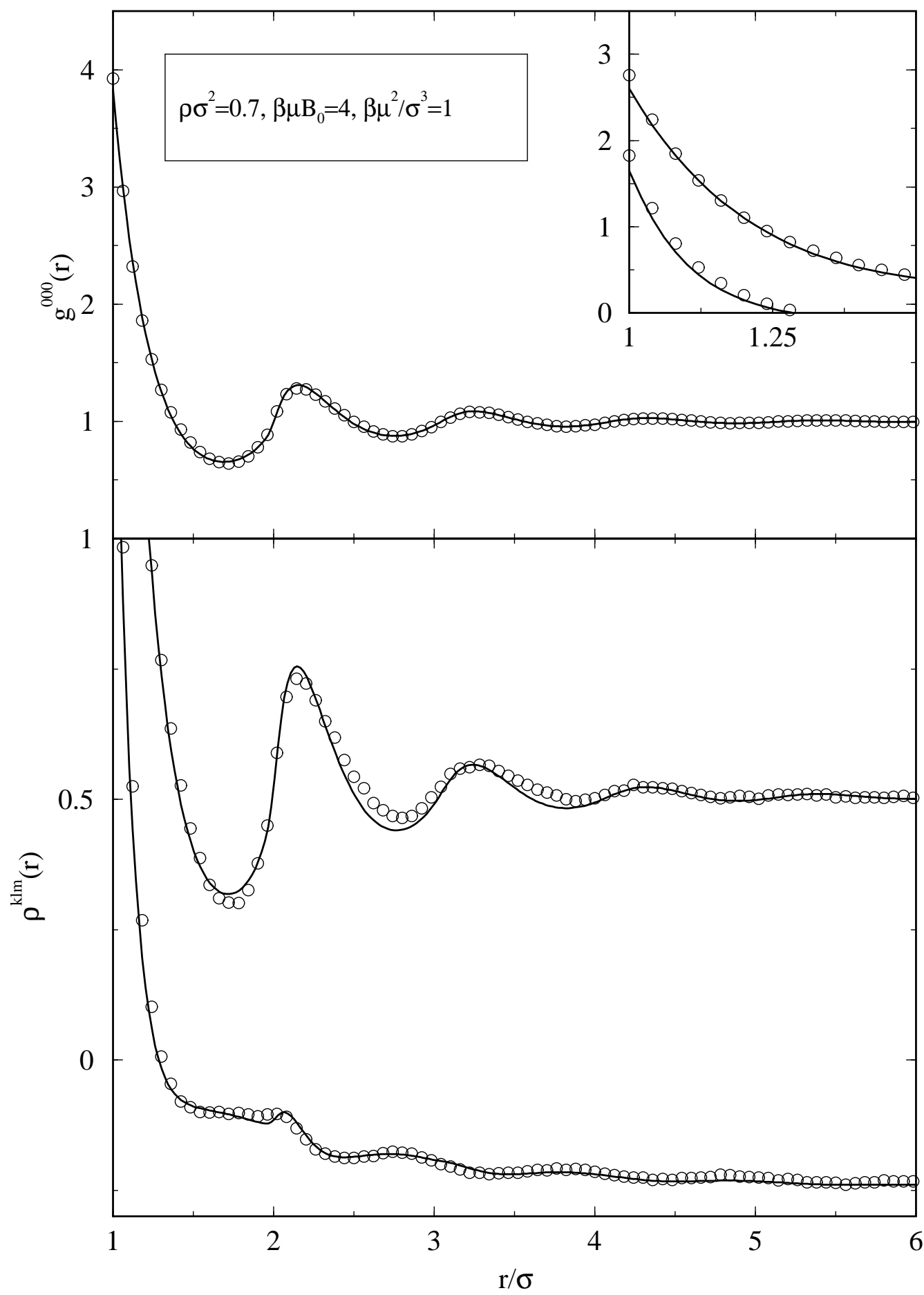

Figure 5. Radial projection $g^{000}(r)$ and angular projections $\rho_{(2)}^{k l m}(r)=\rho_{(2)}^{110}(r)$ $\& \rho_{(2)}^{112}(r) / \rho^{2}$ of the normalized pair density function $\rho_{(2)}(12) / \rho^{2}$ in a ferrofluid monolayer of dipolar hard spheres at $\rho \sigma^{2}=0.7$ and $\beta \mu B_{0}=4$. Here and in figure 6 the angular projection curve with the largest contact value corresponds to $\rho_{(2)}^{112}(r)$ for $\beta \mu B_{0}=0$ and $\rho_{(2)}^{110}(r)$ for $\beta \mu B_{0}=4$. Open circles denote MC data and solid lines represent RHNC results. 


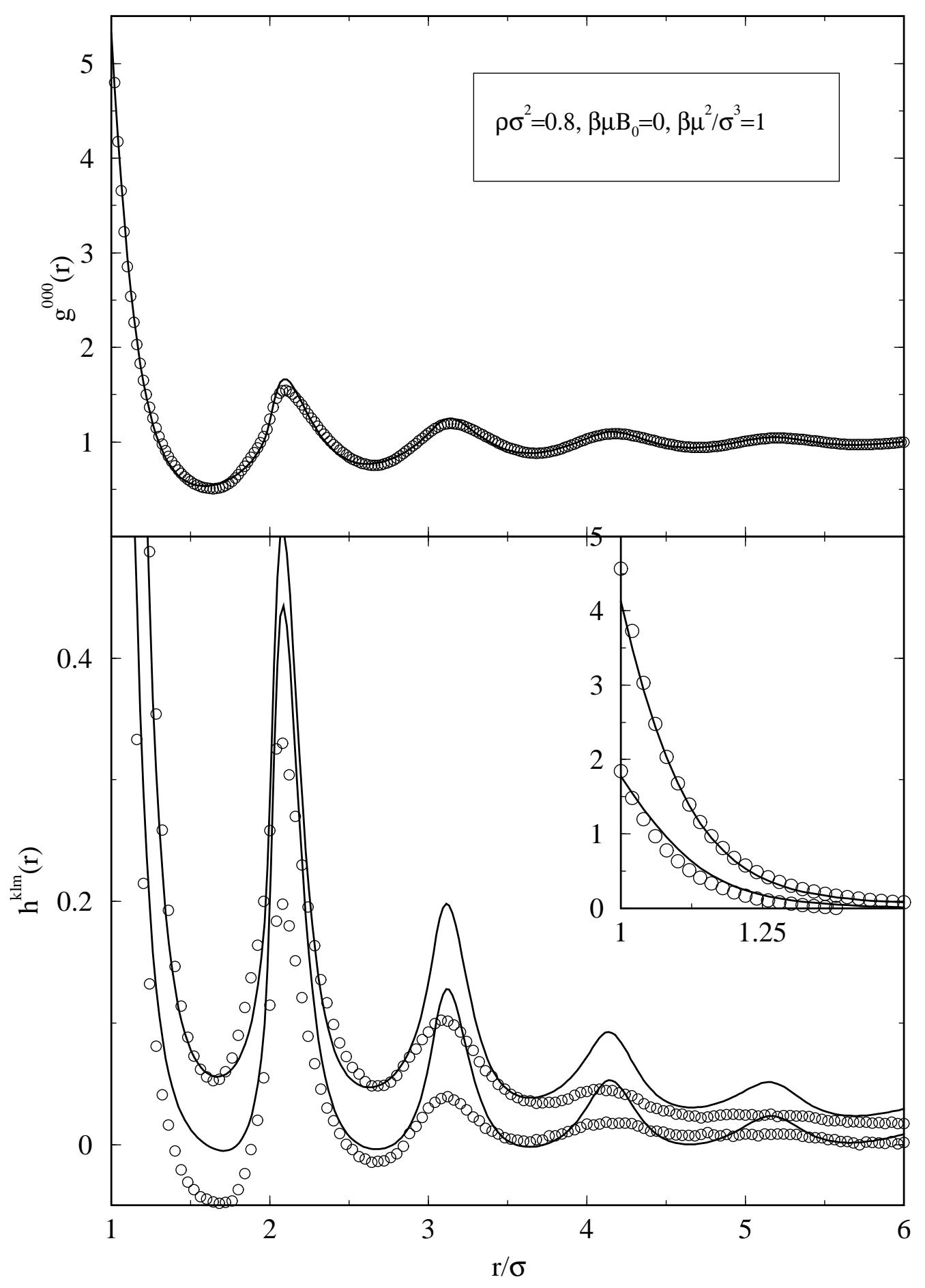

Figure 6. Same as figure 5 for $\rho \sigma^{2}=0.8$ and $\beta \mu B_{0}=0$. 


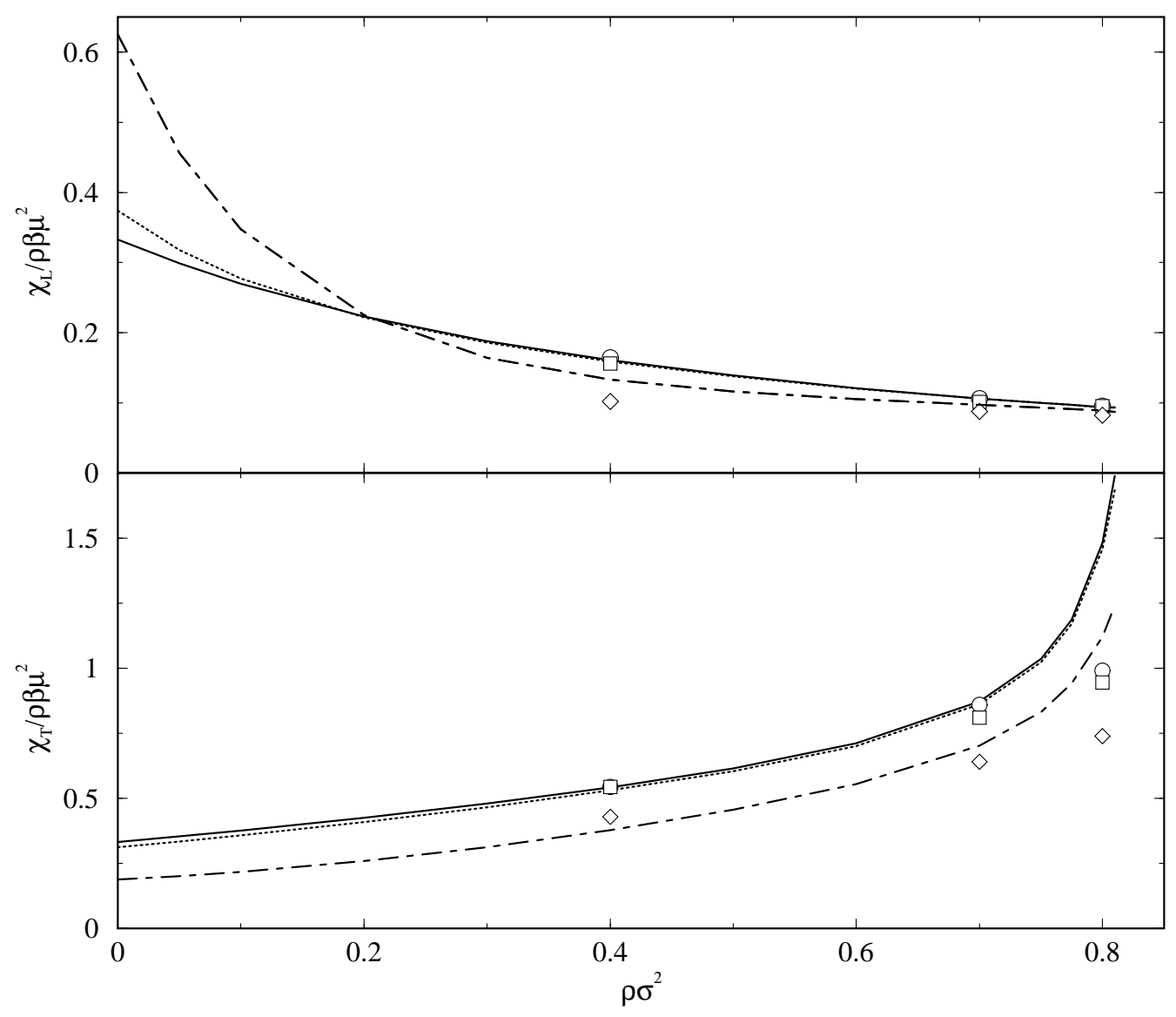

Figure 7. Longitudinal and transverse magnetic susceptibilities of a ferrofluid monolayer in the RHNC approximation (lines) and from MC simulation (symbols). Solid lines (circles) correspond to $\beta \mu B_{0}=0$, dotted lines (squares) to $\beta \mu B_{0}=1$, and dash-dotted lines (diamonds) to $\beta \mu B_{0}=4$. The error on the $\mathrm{MC}$ results for the susceptibilities is about $5 \%$. 

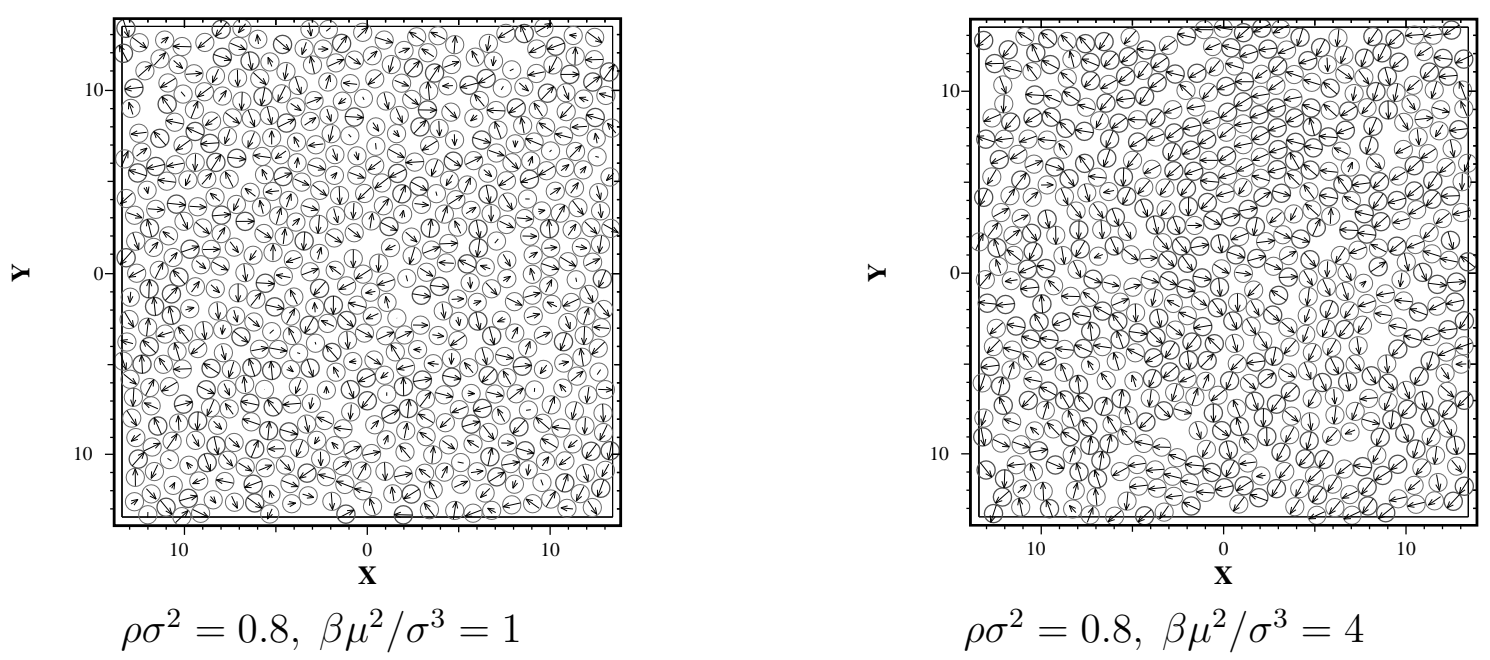

Figure 8. Planar projection of simulation snapshots of a ferrofluid monolayer for two different dipole moments without external field. The arrows indicate the projection of the three dimensional magnetic dipole.

it could be the case that the ordering process for a low dipole moment in a finite system is an extremely slow one that is only captured by the simulation at lower temperatures, although calculations for various systems sizes and lengths of the run do not seem to support this possibility. The situation thus remains inconclusive, though we are of the opinion that the lack of convergence in the integral equation procedure is indicating the onset of some in-plane ordering.

In summary, in this contribution, it has been shown that the inhomogeneous Ornstein-Zernike relation, coupled with a one body relation, either BG or Lovett's equation, is a powerful tool to study orientational transitions and orientationally ordered phases in a wide range of systems and physical conditions. Still, a more thorough comparison between different one body closures remains to be done and our information on the antiferromagnetic system is far from complete.

\section{Acknowledgments}

E.L. thanks the Dirección General de Enseñanza Superior e Investigación Científica (Spain) for support of this work under Grant No. PB97-0258-C02-02.

\section{References}

1. Rosensweig R.E. Ferrohydrodynamics. Cambridge, Cambridge University, 1985.

2. Albrecht T., Bührer C., Fähnle M., Maier K., Platzek D., Reske J. // Appl. Phys. A, 1997, vol. 65, p. 215.

3. Bush G., Guentherodt H.J. // Phys. Lett., 1968, vol. 27A, p. 110; Kraeft B., Alexander H. // Phys. Konden. Mater., 1973, vol. 16, p. 281. 
4. Taylor M.B., Gyorffy B.L. // J. Phys.: Condens. Matter, 1993, vol. 5, p. 4527.

5. Zahn K., Méndez-Alcaraz J.M., Maret G. // Phys. Rev. Lett., 1997, vol. 79, p. 175.

6. Zahn K., Lenke R., Maret G. // Phys. Rev. Lett., 1999, vol. 82, p. 2721.

7. Henderson D. - In: Fundamentals of Inhomogeneous Fluids. Edited by Henderson D., New York, Dekker, 1992, chap. 4.

8. Kinoshita M., Harada M. // Mol. Phys., 1993, vol. 79, p. 145.

9. Wei D., Patey G.N., Perera A. // Phys. Rev. E, 1993, vol. 47, p. 506.

10. Lomba E., Weis J.J., Almarza N.G., Bresme F., Stell G. // Phys. Rev. E., 1994, vol. 49, p. 5169.

11. Lomba E., Weis J.J., Stell G. // 1994, vol. 50, p. 3853.

12. Weis J.J., Nijmeijer M.J.D., Tavares J.M., Telo da Gama M.M. // Phys. Rev. E, 1997, vol. 55 , p. 456.

13. Nijmeijer M.J.P., Weis J.J. // Phys. Rev. Lett., 1995, vol. 75, p. 2887; Phys. Rev. E, 1996, vol. 53, p. 591.

14. Weis J.J., Levesque D., Zarragoicoechea G.J. // Phys. Rev. Lett., 1992, vol. 69, p. 913.

15. Perera A., Kusalik P.G., Patey G.N. // J. Chem. Phys., 1987, vol. 87, p. 1295.

16. Schinagl F., Iro H., Folk R. // Eur. Phys. J. B, 1999, vol. 8, p. 113.

17. Sokolovska T.G. // Physica A, 1998, vol. 253, p. 459.

18. Lovett R.A., Mou C.Y., Buff F.P. // J. Chem. Phys., 1976, vol. 65, p. 570.

19. Lado F., Lomba E. // Phys. Rev. Lett, 1998, vol. 80, p. 3535.

20. Zhong H., Petschek R.G. // Phys. Rev. E, 1995, vol. 51, p. 2263; ibid., 1996, vol. 53, p. 4944.

21. Holovko M.F., Sokolovska T.G. // J. Mol. Liq., 1999, vol. 82, p. 161.

22. Lado F., Lomba E., Weis J.J. // Phys. Rev. E, 1998, vol. 58, p. 3478.

23. Perera A. // Phys. Rev. E, 1999, vol. 60, p. 2912.

24. Lado F. // Phys. Rev. E, 1997, vol. 55, p. 426.

25. Klapp S.H.L., Patey G.N. // J. Chem. Phys., 2000, vol. 112, p. 3832.

26. Lomba E., Lado F., Weis J.J. // Phys. Rev. E, 2000, vol. 61, p. 3838.

27. Lomba E., Lado F. (unpublished results).

28. Bogolugov N.N. Selected Papers. Kyiv, Naukova Dumka, 1971, vol. 3, p. 174

29. Lado F. // Phys. Rev. A, 1973, vol. 8, p. 2548.

30. Zerah G., Hansen J.P. // J. Chem. Phys., 1986, vol. 84, p. 2336.

31. Lado F. // Mol. Phys., 1982, vol. 47, p. 283.

32. Holm C., Janke W. // Phys. Rev. B, 1993, vol. 48, p. 936.

33. Chen K., Ferrenberg A.M., Landau D.P. // Phys. Rev. B, 1993, vol. 48, p. 3249.

34. Stevens M.J., Grest G.S. // Phys. Rev. E, 1995, vol. 51, p. 5962. 


\title{
Підхід інтегральних рівнянь до орієнтаційних фазових переходів у дво- і тривимірних невпорядкованих системах
}

\author{
Е.Ломба ${ }^{1}$, Ф.Ладо ${ }^{2}$, Дж.Дж.Вейс ${ }^{3}$ \\ 1 Інститут фізичної хімії \\ Серрано 119, E-28006 Мадрід, Іспанія \\ 2 Державний університет північної Кароліни, \\ 27695-8202, США \\ 3 Лабораторія теоретичної фізики, \\ Паризький університет, 91405 Орсей, Франція
}

Отримано 1 серпня 2000 р.

Використання неоднорідних рівнянь Орнштейна-Церніке для вивчення фазових переходів і впорядкованих фаз в магнітних системах досліджується як у невпорядкованих гайзенбергівських системах так і в простій моделі для двовимірного ферофлюїдного моношару. Неоднорідне рівняння Орнштейна-Церніке, крім таких замикань як середньосферичне, гіперланцюгове і наближення Зера-Гансена, мусить бути доповнене одно-частинковим замиканням, для якого було використано в цій статті рівняння Борна-Гріна. Отримані результати доводять, що запропонований підхід може давати точні оцінки для переходу парамагнетик-феромагнетик в тривимірному гайзенбергівському спіновому флюїді, надійно відтворюючи структуру ізотропної і впорядкованої фаз. У двох вимірах, результати є, безумовно, точними як для дипольної плівки без поля, так і в присутності зовнішніх перпендикулярно направлених полів. При високих густинах/дипольних моментах рівняння передбачають перехід до фази, в якій диполі лежать в основному в площині і утворюють вихороподібні структури. Наявність цієї нової фази є знайдена при дещо сильніших параметрах при моделюванні.

Ключові слова: інтегральні рівняння, феромагнітні переходи, антиферомагнетизм, дипольні плівки

PACS: 61.20.Gy, 68.15.+e, 75.10.-b, 75.30.-m 